\title{
Incidence of bone metastases in patients with solid tumors: analysis of oncology electronic medical records in the United States
}

Rohini K. Hernandez ${ }^{1}$, Sally W. Wade ${ }^{2}$, Adam Reich ${ }^{3}$, Melissa Pirolli ${ }^{3}$, Alexander Liede ${ }^{5^{*}}$ and Gary H. Lyman ${ }^{4}$

\begin{abstract}
Background: Bone metastases commonly occur in conjunction with solid tumors, and are associated with serious bone complications. Population-based estimates of bone metastasis incidence are limited, often based on autopsy data, and may not reflect current treatment patterns.

Methods: Electronic medical records (OSCER, Oncology Services Comprehensive Electronic Records, 569,000 patients, 52 US cancer centers) were used to identify patients $\geq 18$ years with a solid tumor diagnosis recorded between 1/1/2004 and 12/31/2013, excluding patients with hematologic tumors or multiple primaries. Each patient's index date was set to the date of his or her first solid tumor diagnosis in the selection period. KaplanMeier analyses were used to quantify the cumulative incidence of bone metastasis with follow-up for each patient from the index date to the earliest of the following events: last clinic visit in the OSCER database, occurrence of a new primary tumor or bone metastasis, end of study (12/31/2014). Incidence estimates and associated 95\% confidence intervals $(\mathrm{Cl})$ are provided for up to 10 years of follow-up for all tumor types combined and stratified by tumor type and stage at diagnosis.
\end{abstract}

Results: Among 382,733 study patients (mean age 64 years; mean follow-up 940 days), breast (36\%), lung (16), and colorectal (12\%) tumors were most common. Mean time to bone metastasis was 400 days (1.1 years). Cumulative incidence of bone metastasis was 2.9\% (2.9-3.0) at 30 days, 4.8\% (4.7-4.8) at one year, 5.6\% (5.5-5.6) at two years, 6. $9 \%(6.8-7.0)$ at five years, and $8.4 \%(8.3-8.5)$ at ten years. Incidence varied substantially by tumor type with prostate cancer patients at highest risk (18\% - 29\%) followed by lung, renal or breast cancer. Cumulative incidence of bone metastasis increased by stage at diagnosis, with markedly higher incidence among patients diagnosed at Stage IV of whom $11 \%$ had bone metastases diagnosed within 30 days.

Conclusions: These estimates of bone metastasis incidence represent the experience of a population with longer follow-up than previously published, and represent experience in the recent treatment landscape. Underestimation is possible given reliance on coded diagnoses but the clinical detail available in electronic medical records contributes to the accuracy of these estimates.

Keywords: Solid tumor, Bone metastasis, Incidence, Epidemiology

\footnotetext{
* Correspondence: aliede@amgen.com

${ }^{5}$ Amgen, Inc., 1120 Veterans Blvd, South San Francisco, CA 94114, USA

Full list of author information is available at the end of the article
} 


\section{Background}

Solid tumors frequently metastasize to bone [1, 2], and these bone metastases are associated with shortened survival [3-7] and increased risk of serious bone complications during the patients' remaining lifespan $[3,8,9]$. Greater bone remodeling coincident with increased osteoblast and osteoclast activity at the site of the bone metastasis are hypothesized to create an environment that symbiotically supports tumor growth and bone destruction, and contributes to the risk of skeletal-related events (SREs) including pathological fractures and spinal cord compressions requiring palliative radiotherapy or surgery to bone $[10,11]$. Patients with an SRE are significantly more likely to experience a subsequent SRE, often have a poorer prognosis and shorter overall survival than patients without an SRE, experience impaired quality of life, including ongoing pain, and consume significantly more health resources compared with patients without SREs [12-14].

Despite the important clinical and economic consequences of bone metastases, the incidence of bone metastases is not well understood as population-based estimates are limited in number and scope, often providing insights for a single tumor type or age group [4-6], or providing estimates on the basis of autopsy data that likely exceed the incidence of bone metastases that are formally diagnosed in routine patient care [15]. In addition, published estimates based on older data may not reflect survival trends under recent treatment advances [16], and long follow-up periods are also rarely reported in the literature.

The current study was conducted to estimate the incidence of bone metastases reflecting the more recent treatment landscape for patients with solid tumors. Specifically, we estimated the cumulative incidence proportion of clinically-identified bone metastases for all solid tumors combined and by tumor type using electronic medical records (EMR) data from oncology clinics in the United States (US). Results are presented for various time intervals during up to ten years of follow-up.

\section{Methods}

Electronic medical records housed in the Oncology Services Comprehensive Electronic Records (OSCER) database were used to identify patients for this study. OSCER contains data from over 569,000 patients treated at 52 geographically-dispersed community and hospital-affiliated oncology practices in the US since 2004. This source population includes patients with health benefits through, Medicare, Medicaid, or commercial coverage, as well as patients who pay directly for their medical care. The Institutional Review Board of each oncology practice approved collaboration to contribute data to a large longitudinal electronic health records database; informed patient consent was waived per the US framework for retrospective noninterventional studies. Individual patient-level data were protected against breach of confidentiality consistent with the final Health Insurance Portability and Accountability Act (HIPAA) Security Rule from the US Department of Health and Human Services.

Patients included in the study population were at least 18 years old and a solid tumor diagnosis recorded between January 1, 2004 and December 31, 2013. Each patient's index date was set to the date of his or her first solid tumor diagnosis during the patient selection period. This date represents the date of the definitive solid tumor diagnosis recorded in the electronic medical record at the patient's oncologist's office. Since most solid tumor patients will initiate their anti-cancer treatments with an oncologists, these are likely to be newly-treated patients.

As we sought to accurately assign patients to a primary solid tumor type based on the available data, we noted that a small percentage $(2.3 \%)$ of patients had more than one primary tumor type recorded within 30 days of the index date. Therefore, the following rules were applied. Patients with multiple synchronous primaries (i.e., 3 or more different tumor types, including the index tumor) within 30 days of the index date were excluded. The following rules were used to determine the primary tumor type for patients with a second tumor type recorded within 30 days of the index tumor. Lung, liver, brain and bone tumors were considered to be metastases of the index tumor. Whenever present, melanoma was considered the primary solid tumor diagnosis. When both a non-specific and a specific tumor diagnosis code were present, the specific diagnosis defined the primary tumor type (e.g., gynecological cancer [non-specific] versus ovarian cancer [specific]). If two specific but different tumor types were recorded (including the index tumor diagnosis), the patient was excluded as the primary solid tumor type could not be clearly distinguished (e.g., breast cancer and colorectal cancer). Patients with only a non-specific tumor type in conjunction with a bone cancer diagnosis were also excluded. Since the primary study outcome was incident bone metastases (ICD-9 diagnosis code 198.5), patients with evidence of bone metastases more than 30 days prior to their index date were also excluded. Patients with bone metastases diagnosed within 30 days before their index date were considered to have bone metastasis at their initial solid tumor diagnosis, and the bone metastasis date was recoded to the index date.

Kaplan-Meier analyses were used to quantify the cumulative incidence of bone metastasis with follow-up for each patient from the index date to the earliest of the following events: last clinic visit in the OSCER database, occurrence 
of bone metastasis (including those diagnosed at index) or a new primary tumor, end of study (December 31, 2014). Incidence estimates and associated 95\% confidence intervals $(\mathrm{CI})$ are provided for up to 10 years of follow-up, with results for all tumor types combined and stratified by tumor type and stage at diagnosis.

\section{Results}

The majority (98\%) of the 390,935 patients identified in OSCER with a new solid tumor diagnosis between January 1, 2004 and December 31, 2013 met all other selection criteria for inclusion in the study (Fig. 1). The most common reasons for exclusion were presence of non-solid tumor diagnoses $(0.7 \%)$ and inability to determine the primary tumor type among patients with an additional tumor type recorded within 30 days prior to the index tumor as per the rules described in the methods section (1\%).

Among the 382,733 study patients (mean age 64 years; mean follow-up 940 days), breast (36\%), lung (16), and colorectal $(12 \%)$ tumors were the most common index tumor types (Table 1). The number of patients identified in each year of the study increased from 16,525 in 2004 to 52,534 in 2013 , with slightly more than half of study patients identified between 2010 and the end of 2013; this trend reflects the growing number of patients in the OSCER database in general.

Of the full study population, 26,250 (6.9\%) patients were diagnosed with bone metastases at index and during follow-up (median follow-up of 548 days [1.5 years] after the index solid tumor diagnosis). The mean time to

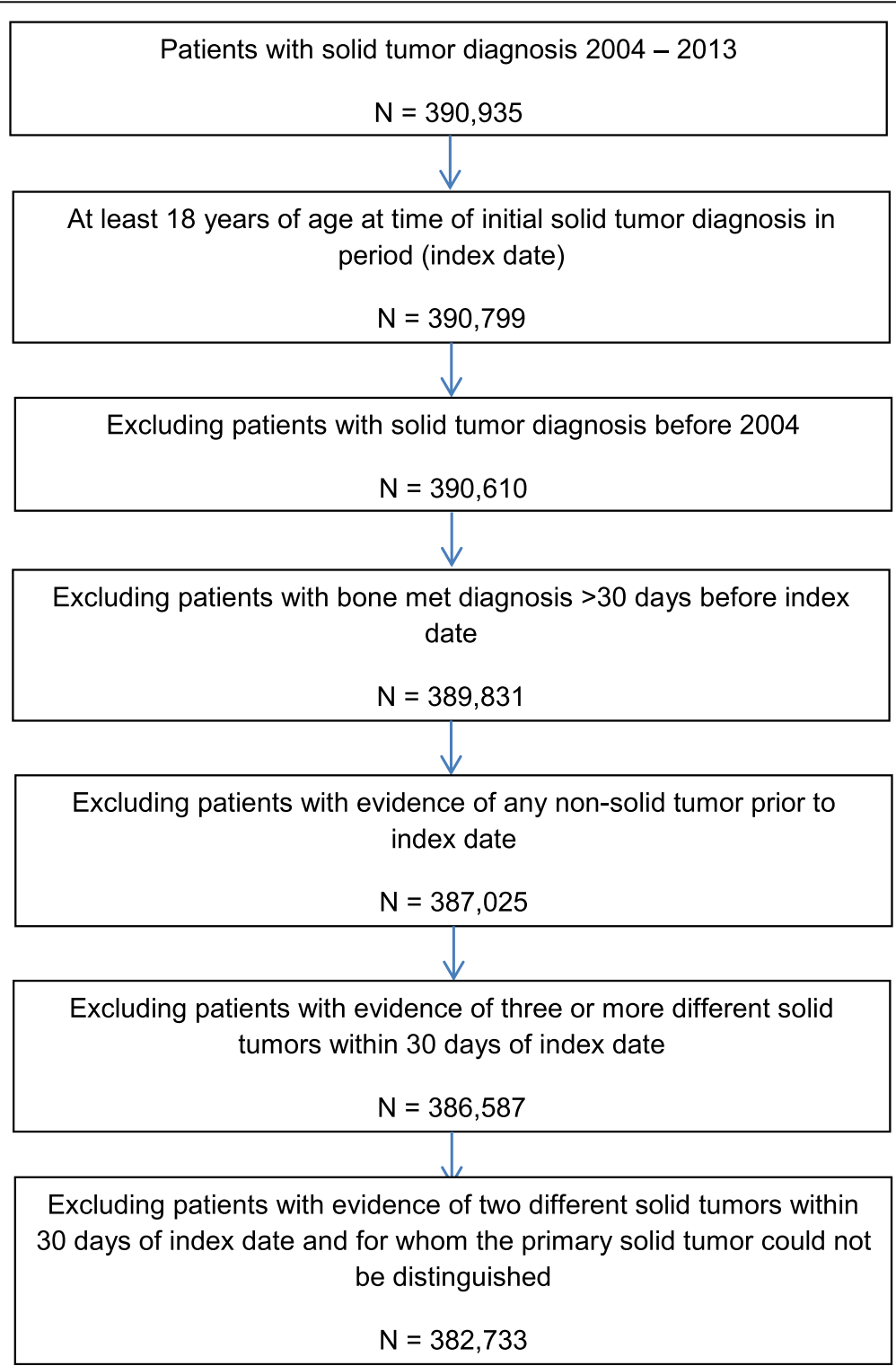

Fig. 1 Selection of Study Patients 


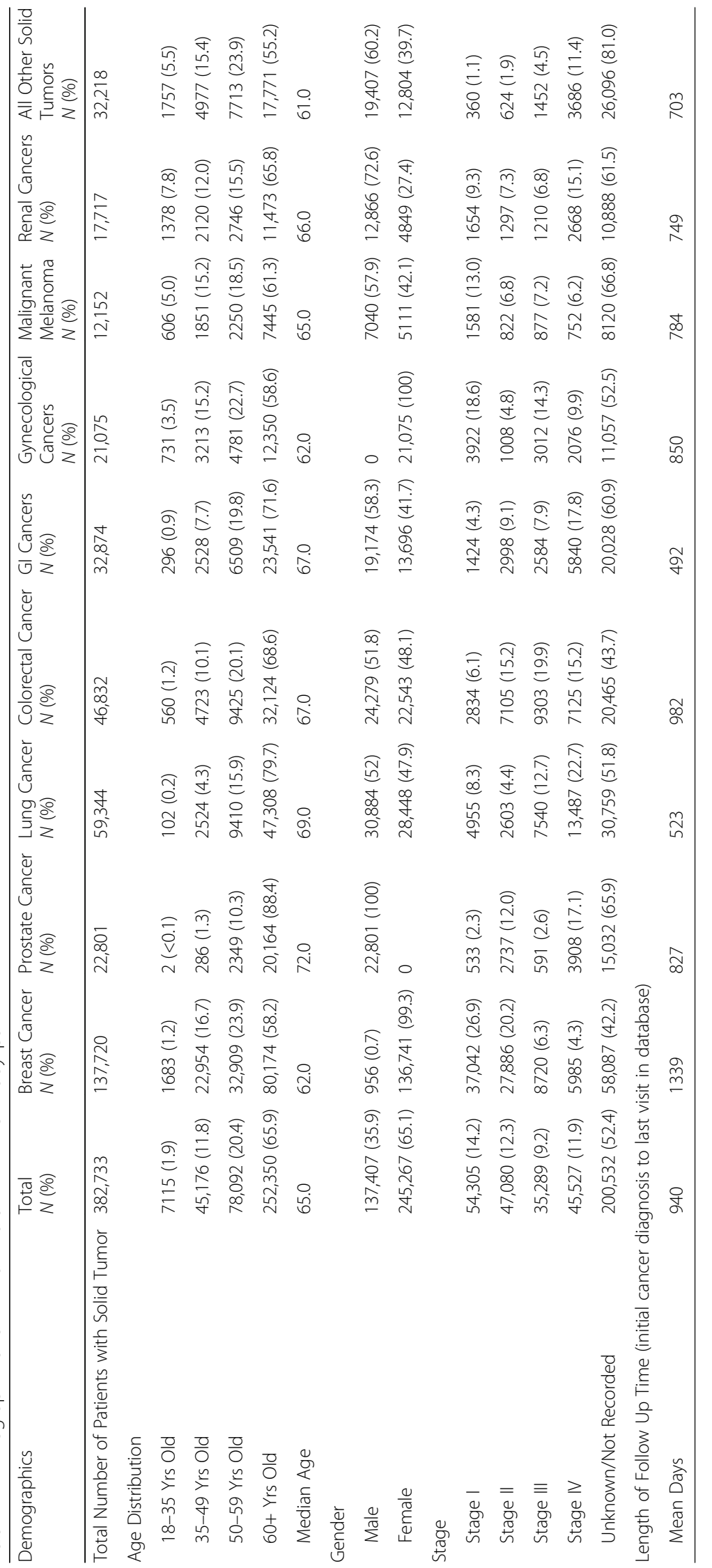


bone metastasis from solid tumor diagnosis was 400 days (1.1 years), while the median time between diagnosis and bone metastasis was 69 days The corresponding intervals were 535 and 226 days after excluding patients with bone metastasis at index, and 700 and 407 days after excluding patients with bone metastasis within 30 days of their primary tumor diagnosis.

For all tumor types combined, the cumulative incidence of bone metastasis (95\% CI) was 2.9\% (2.9-3.0) at 30 days post-index date, $4.8 \%(4.7-4.8)$ at one year, $5.6 \%$ $(5.5-5.6)$ at two years, $6.9 \%(6.8-7.0)$ at five years, and $8.4 \%(8.3-8.5)$ at ten years (Fig. 2, Table 2). Bone metastasis incidence was highly variable depending on the primary tumor type, with prostate cancer patients at highest risk of developing bone metastases, followed by patients with lung, renal or breast cancer (Fig. 2, Table 2). The largest increase in incidence over the ten year follow-up was observed among patients with prostate cancer.

The cumulative incidence of bone metastasis increased by stage at diagnosis, for the population overall (Fig. 3) and each tumor type (Table 3), with this pattern appearing in each follow-up interval assessed. In every case, the incidence of bone metastasis among patients diagnosed at Stage IV was markedly higher than incidence among patients diagnosed at less advanced disease stages. Although bone metastases were diagnosed on or within
30 days of the solid tumor diagnosis in 11\% (5206/ 45,527 ) of the patients who were diagnosed at Stage IV, the cumulative incidence of bone metastasis continued to increase in these late-stage patients over time, regardless of tumor type.

\section{Discussion}

This study estimated the cumulative incidence of bone metastasis among patients with solid tumors using real world electronic medical record data from oncology practices in the US. To our knowledge, this is the first largescale US study to estimate the incidence of bone metastases for all solid tumors combined and by tumor type, with patients followed for up to 10 years after their initial solid tumor diagnosis. Cumulative incidence increased from $2.9 \%$ within 30 days of the first solid tumor diagnosis in the study period to $8.4 \%$ during a ten year follow-up period. Bone metastasis incidence increased most quickly in the first two years for the solid tumor population as a whole, with the most common tumor types also showing the greatest increases in incidence in the first year or two post-diagnosis. The availability of long-term follow-up data for the study population allowed us to determine that the cumulative incidence of bone metastasis also continued increasing for at least ten years after the initial solid tumor diagnosis, regardless of tumor type.

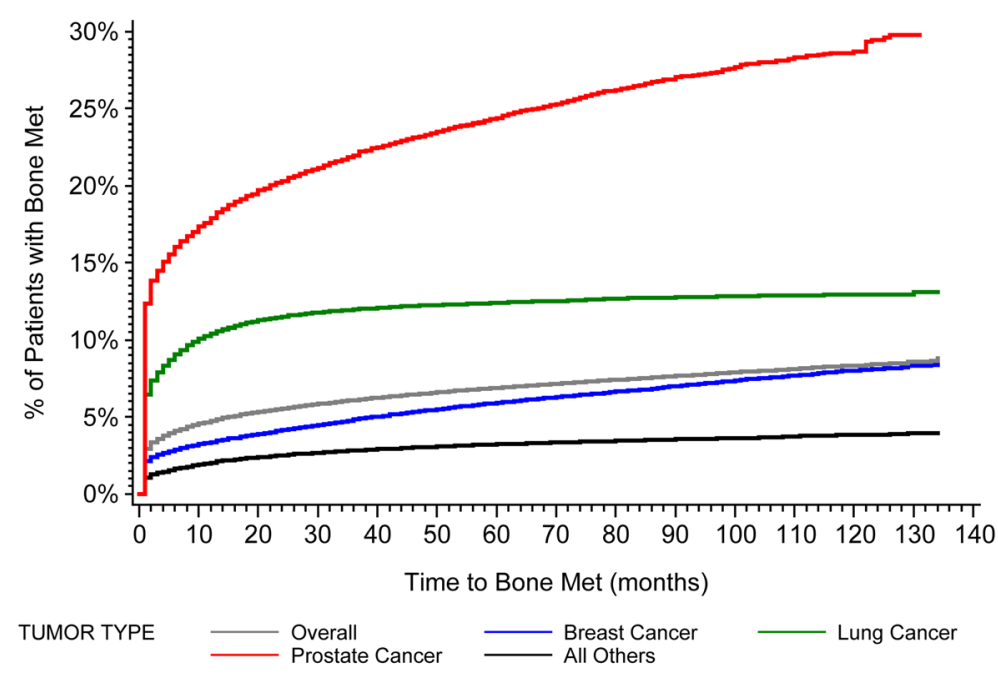

\begin{tabular}{|lcccc|}
\hline & \multicolumn{4}{c|}{ Number of Patients with Eligible Follow Up } \\
\cline { 2 - 5 } Tumor Type & 1 year & 2 years & 5 years & 10 years \\
\hline All tumor types combined $(\mathrm{N}=382,733)$ & $(\mathrm{N}=382,733)$ & $(\mathrm{N}=337,040)$ & $(\mathrm{N}=202,637)$ & $(\mathrm{N}=43,665)$ \\
Breast $(\mathrm{N}=137,720)$ & $(\mathrm{N}=137,720)$ & $\mathrm{N}=123,008)$ & $(\mathrm{N}=77,525)$ & $(\mathrm{N}=17,204)$ \\
Prostate $(\mathrm{N}=22,801)$ & $(\mathrm{N}=22,801)$ & $(\mathrm{N}=20,492)$ & $(\mathrm{N}=14,040)$ & $(\mathrm{N}=6,156)$ \\
Lung $(\mathrm{N}=59,344)$ & $(\mathrm{N}=59,344)$ & $(\mathrm{N}=52,027)$ & $(\mathrm{N}=31,381)$ & $(\mathrm{N}=7,351)$ \\
$\begin{array}{l}\text { All other tumors except breast, } \\
\text { prostate and lung }(\mathrm{N}=162,868)\end{array}$ & $(\mathrm{N}=162,868)$ & $(\mathrm{N}=141,513)$ & $(\mathrm{N}=79,691)$ & $(\mathrm{N}=12,954)$ \\
\hline
\end{tabular}

Fig. 2 Cumulative bone metastasis incidence in 10-year follow-up of patients with solid tumors 
Table 2 1-, 2-, 5-, and 10-year incidence of bone metastases by tumor type

\begin{tabular}{|c|c|c|c|c|}
\hline \multirow[t]{2}{*}{ Tumor type } & \multicolumn{4}{|c|}{ Incidence of bone metastases (\%) } \\
\hline & 1 -year $(95 \% \mathrm{Cl})$ & 2-year (95\% Cl) & 5-year $(95 \% \mathrm{Cl})$ & 10-year $(95 \% \mathrm{Cl})$ \\
\hline All tumor types combined $(N=382,733)$ & $4.8(4.7-4.8)$ & $5.6(5.5-5.6)$ & $6.9(6.8-7.0)$ & $8.4(8.3-8.5)$ \\
\hline Breast $(N=137,720)$ & $3.4(3.3-3.5)$ & $4.2(4.1-4.3)$ & $6.0(5.8-6.1)$ & $8.1(7.9-8.3)$ \\
\hline Prostate $(N=22,801)$ & $18.0(17.5-18.5)$ & $20.4(19.9-20.9)$ & $24.5(23.9-25.1)$ & $29.2(28.3-30.1)$ \\
\hline Lung $(N=59,344)$ & $10.4(10.2-10.7)$ & $11.5(11.3-11.8)$ & $12.4(12.1-12.7)$ & $12.9(12.6-13.2)$ \\
\hline Colorectal $(N=46,832)$ & $1.0(0.9-1.1)$ & $1.4(1.3-1.5)$ & $2.1(2.0-2.3)$ & $2.7(2.5-2.9)$ \\
\hline Gastrointestinal $(N=32,874)$ & $2.3(2.1-2.5)$ & $2.7(2.6-2.9)$ & $3.2(3.0-3.4)$ & $3.6(3.3-3.8)$ \\
\hline Gynecological $(N=21,075)$ & $1.1(0.9-1.2)$ & $1.3(1.2-1.5)$ & $1.9(1.7-2.1)$ & $2.4(2.1-2.7)$ \\
\hline Malignant melanoma $(N=12,152)$ & $1.6(1.4-1.8)$ & $2.0(1.7-2.2)$ & $2.5(2.2-2.8)$ & $3.0(2.6-3.4)$ \\
\hline Renal $(N=17,717)$ & $5.8(5.5-6.2)$ & $6.9(6.6-7.3)$ & $8.4(8.0-8.9)$ & $9.9(9.3-10.5)$ \\
\hline All other tumors $(N=32,218)$ & $2.0(1.8-2.1)$ & $2.5(2.3-2.7)$ & $3.2(3.0-3.4)$ & $3.9(3.5-4.2)$ \\
\hline
\end{tabular}

In our study population, patients with prostate tumors exhibited markedly higher incidence of bone metastases in every time interval assessed, and substantially larger increases in incidence from the first through tenth year of follow-up. It is important to note that the sample of prostate cancer patients with data in OSCER includes only patients who were treated at a participating oncology clinic. This approach may bias our sample toward men with later stage disease, compared with the general prostate cancer population, by excluding men who received their prostate cancer care in urology clinics. These excluded patients would presumably be more likely to have early stage disease and a generally lower propensity for disease progression including the development of bone metastases over time. If early stage patients are under-represented in our sample, as we expect, our results likely exceed the true bone metastasis incidence in a more typical prostate cancer population. Although early stage prostate cancer patients may be less wellrepresented in our population, the observed trend in

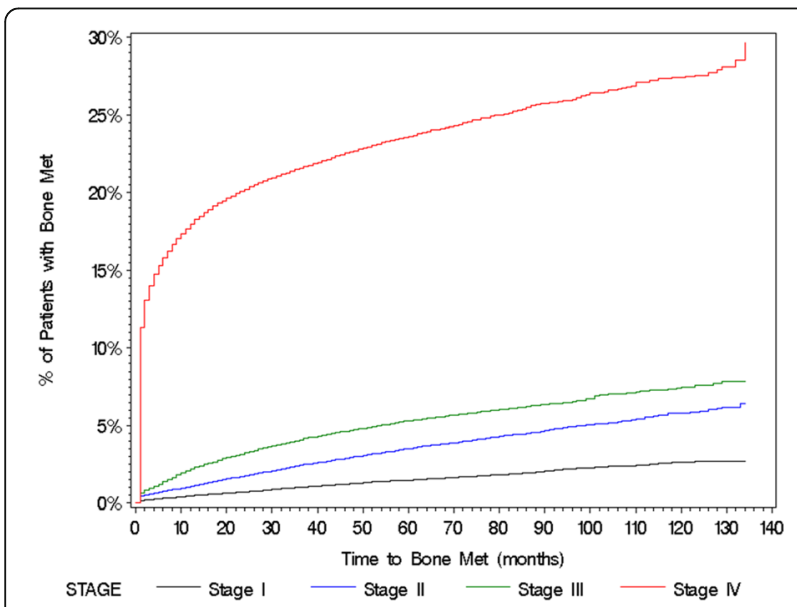

Fig. 3 Cumulative bone metastasis incidence by stage at diagnosis for all solid tumors combined incidence over time suggests that ongoing monitoring of bone health may continue to be important for patients with prostate cancer, even years after the initial prostate cancer diagnosis. Surprisingly, the literature suggests that such monitoring to identify an initial bone metastasis is not generally routine with one study reporting that even prostate cancer patients at high risk of developing bone metastases, such as those with prostate-specific antigen doubling time less than 3 months, did not routinely receive a second bone scan within one year after a first negative bone scan [17]. There is not yet a universal guideline regarding imaging of men with M0 castrationresistant prostate cancer, but appropriate screening frequency will need to balance the potential benefits that could be obtained through early detection and treatment with cost considerations [17].

Not surprisingly, we found that the incidence of bone metastasis was higher among patients with more advanced disease (i.e., higher stage) at diagnosis in the solid tumor population overall and for the individual tumor types that we examined. This pattern continued over time; we noted this relationship between stage at diagnosis and bone metastasis incidence in every follow-up interval for the study population overall and for each tumor type. Greater incidence of bone metastases among patients with higher cancer stages at diagnosis has also been reported previously in population-based studies of breast cancer patients in Denmark and the United Kingdom (UK) $[16,18]$.

The literature on bone metastasis incidence in the US provides estimates for three important tumor types, but only for individuals with Medicare coverage whose administrative claims data could be linked to data in the population-based Surveillance Epidemiology and End Results (SEER) cancer registry [4-6]. Using these data, Sathiakumar et al. have reported separately on the experience of patients diagnosed with lung, breast or prostate cancer between 1999 and 2005 and followed through the end of study in 2006. These studies were 
Table 3 1-, 2-, 5-, and 10-year incidence of bone metastases by tumor type and stage at diagnosis

\begin{tabular}{|c|c|c|c|c|}
\hline \multirow[t]{2}{*}{ Tumor type } & \multicolumn{4}{|c|}{ Incidence of bone metastases (\%) } \\
\hline & 1 -year $(95 \% \mathrm{Cl})$ & 2-year (95\% Cl) & 5-year $(95 \% \mathrm{Cl})$ & 10-year (95\% Cl) \\
\hline \multicolumn{5}{|c|}{ All tumor types combined $(\mathrm{N}=382,733)$} \\
\hline Stage I $(N=54,305)$ & $0.5(0.4-0.5)$ & $0.7(0.7-0.8)$ & $1.5(1.4-1.6)$ & $2.7(2.4-2.9)$ \\
\hline Stage $\|(N=47,080)$ & $1.1(1.0-1.2)$ & $1.8(1.7-1.9)$ & $3.6(3.4-3.8)$ & $5.9(5.6-6.3)$ \\
\hline Stage III $(N=35,289)$ & $2.2(2.0-2.3)$ & $3.3(3.1-3.5)$ & $5.4(5.1-5.6)$ & $7.5(7.1-8.0)$ \\
\hline Stage IV $(N=45,527)$ & $18.0(17.7-18.4)$ & $20.3(19.9-20.7)$ & $23.7(23.3-24.1)$ & $27.6(27.0-28.2)$ \\
\hline \multicolumn{5}{|l|}{ Breast $(N=137,720)$} \\
\hline Stage I $(N=37,042)$ & $0.3(0.3-0.4)$ & $0.5(0.5-0.6)$ & $1.2(1.1-1.4)$ & $2.4(2.1-2.7)$ \\
\hline Stage $\|(N=27,886)$ & $1.0(0.9-1.2)$ & $1.8(1.6-1.9)$ & $3.9(3.7-4.2)$ & $6.5(6.0-6.9)$ \\
\hline Stage III $(N=8720)$ & $2.9(2.6-3.3)$ & $4.9(4.4-5.3)$ & $10.1(9.4-10.8)$ & $15.3(14.1-16.5)$ \\
\hline Stage IV $(N=5985)$ & $36.4(35.2-37.7)$ & $41.4(40.2-42.7)$ & $50.6(49.3-52.0)$ & $61.4(59.5-63.2)$ \\
\hline \multicolumn{5}{|l|}{ Prostate $(N=22,801)$} \\
\hline Stage I $(N=533)$ & $3.0(1.8-4.9)$ & $4.4(2.9-6.5)$ & $7.7(5.4-10.8)$ & $12.1(8.3-17.4)$ \\
\hline Stage $\|(N=2737)$ & $3.3(2.6-4.0)$ & $4.1(3.4-4.9)$ & $7.3(6.3-8.4)$ & $15.8(12.0-20.6)$ \\
\hline Stage III $(N=591)$ & $9.0(6.9-11.6)$ & $11.5(9.2-14.4)$ & $16.4(13.4-19.9)$ & $23.4(18.7-29.0)$ \\
\hline Stage IV $(N=3908)$ & $45.3(43.8-46.9)$ & $51.4(49.9-53.0)$ & $61.1(59.5-62.8)$ & $70.7(68.4-72.9)$ \\
\hline \multicolumn{5}{|l|}{ Lung $(N=59,344)$} \\
\hline Stage I $(N=4955)$ & $1.4(1.1-1.8)$ & $2.3(1.9-2.7)$ & $3.6(3.0-4.2)$ & $5.1(4.1-6.4)$ \\
\hline Stage $\|(N=2603)$ & $2.7(2.2-3.4)$ & $4.2(3.4-5.0)$ & $5.6(4.7-6.7)$ & $8.4(6.9-10.4)$ \\
\hline Stage III $(N=7540)$ & $4.4(4.0-4.9)$ & $5.8(5.3-6.4)$ & $6.7(6.2-7.3)$ & $7.4(6.7-8.2)$ \\
\hline Stage IV $(N=13,487)$ & $22.9(22.2-23.6)$ & $24.5(23.8-25.3)$ & $25.8(25.1-26.6)$ & $26.2(25.4-27.0)$ \\
\hline \multicolumn{5}{|l|}{ Colorectal $(N=46,832)$} \\
\hline Stage I $(N=2834)$ & $0.2(0.1-0.5)$ & $0.4(0.2-0.7)$ & $1.0(0.7-1.5)$ & $1.6(1.0-2.6)$ \\
\hline Stage $\|(N=7105)$ & $0.2(0.1-0.3)$ & $0.5(0.3-0.7)$ & $1.0(0.7-1.3)$ & $1.6(1.1-2.2)$ \\
\hline Stage III (N = 9303) & $0.4(0.3-0.5)$ & $0.7(0.6-0.9)$ & $1.5(1.2-1.8)$ & $1.9(1.6-2.3)$ \\
\hline Stage IV $(N=7125)$ & $3.0(2.7-3.5)$ & $4.1(3.7-4.6)$ & $5.8(5.2-6.4)$ & $6.6(5.9-7.4)$ \\
\hline \multicolumn{5}{|c|}{ Gastrointestinal $(N=32,874)$} \\
\hline Stage I $(N=1424)$ & $0.4(0.2-0.9)$ & $0.7(0.3-1.3)$ & $1.5(0.9-2.5)$ & $2.2(1.2-3.9)$ \\
\hline Stage $\|(N=2998)$ & $0.7(0.5-1.1)$ & $1.3(0.9-1.7)$ & $2.2(1.7-2.9)$ & $2.9(2.0-4.1)$ \\
\hline Stage III $(N=2584)$ & $1.2(0.9-1.7)$ & $1.9(1.4-2.5)$ & $2.7(2.1-3.4)$ & $2.9(2.2-3.7)$ \\
\hline Stage IV $(N=5840)$ & $5.3(4.7-5.9)$ & $6.1(5.5-6.8)$ & $6.8(6.2-7.5)$ & $7.4(6.5-8.4)$ \\
\hline \multicolumn{5}{|c|}{ Gynecological $(N=21,075)$} \\
\hline Stage I $(N=3922)$ & $0.2(0.1-0.4)$ & $0.5(0.3-0.8)$ & $0.9(0.6-1.3)$ & $1.9(1.1-3.2)$ \\
\hline Stage $\|(N=1008)$ & $0.6(0.3-1.3)$ & $1.0(0.5-1.9)$ & $1.8(1.1-3.0)$ & $2.0(1.3-3.3)$ \\
\hline Stage III $(N=3012)$ & $0.7(0.4-1.0)$ & $1.2(0.9-1.7)$ & $2.1(1.6-2.8)$ & $3.1(1.9-5.1)$ \\
\hline Stage IV $(N=2076)$ & $4.0(3.3-5.0)$ & $4.7(3.8-5.7)$ & $6.2(5.2-7.5)$ & $7.5(5.9-9.6)$ \\
\hline \multicolumn{5}{|c|}{ Malignant melanoma $(\mathrm{N}=12,152)$} \\
\hline Stage I $(N=1581)$ & $0.2(0.1-0.6)$ & $0.3(0.1-0.7)$ & $0.6(0.3-1.3)$ & $0.9(0.4-1.7)$ \\
\hline Stage $\|(N=822)$ & $0.7(0.3-1.6)$ & $1.1(0.6-2.1)$ & $1.9(1.1-3.3)$ & $1.9(1.1-3.3)$ \\
\hline Stage III $(N=877)$ & $0.6(0.2-1.4)$ & $1.2(0.6-2.1)$ & $1.2(0.6-2.1)$ & $1.2(0.6-2.1)$ \\
\hline Stage IV $(N=752)$ & $6.1(4.6-8.1)$ & $7.4(5.7-9.5)$ & $9.0(7.1-11.5)$ & $10.4(8.0-13.5)$ \\
\hline \multicolumn{5}{|l|}{ Renal $(N=17,717)$} \\
\hline Stage I $(N=1654)$ & $0.8(0.5-1.4)$ & $1.1(0.7-1.8)$ & $2.4(1.7-3.4)$ & $5.1(3.4-7.6)$ \\
\hline Stage $\|(N=1297)$ & $1.5(1.0-2.4)$ & $2.4(1.7-3.4)$ & $3.9(2.9-5.3)$ & $5.0(3.5-7.1)$ \\
\hline
\end{tabular}


Table 3 1-, 2-, 5-, and 10-year incidence of bone metastases by tumor type and stage at diagnosis (Continued)

\begin{tabular}{|c|c|c|c|c|}
\hline \multirow[t]{2}{*}{ Tumor type } & \multicolumn{4}{|c|}{ Incidence of bone metastases (\%) } \\
\hline & 1-year (95\% Cl) & 2-year $(95 \% \mathrm{Cl})$ & 5-year (95\% Cl) & 10-year $(95 \% \mathrm{Cl})$ \\
\hline Stage III $(N=1210)$ & $2.1(1.5-3.1)$ & $3.3(2.4-4.5)$ & $5.0(3.8-6.5)$ & $6.8(5.1-9.1)$ \\
\hline Stage IV $(N=2668)$ & $15.5(14.2-16.9)$ & $18.3(16.9-19.8)$ & $22.3(20.7-24.1)$ & $26.2(23.6-29.0)$ \\
\hline \multicolumn{5}{|c|}{ Other Tumors $(N=162,868)$} \\
\hline Stage I $(N=360)$ & $0.8(0.3-2.6)$ & $1.7(0.8-3.7)$ & $2.1(1.0-4.4)$ & $5.2(1.6-16.3)$ \\
\hline Stage $\|(N=624)$ & $0.8(0.3-1.9)$ & $1.3(0.7-2.6)$ & $3.2(2.0-5.3)$ & $4.8(2.9-7.8)$ \\
\hline Stage III $(N=1452)$ & $0.8(0.4-1.4)$ & $1.5(1.0-2.3)$ & $1.9(1.3-2.8)$ & $3.5(2.1-5.7)$ \\
\hline Stage IV $(N=3686)$ & $2.7(2.2-3.3)$ & $3.8(3.2-4.5)$ & $4.6(4.0-5.4)$ & $5.3(4.3-6.4)$ \\
\hline
\end{tabular}

limited to patients age 65 and older and to those individuals who had full fee-for-service Medicare coverage for at least 6 months prior to their cancer diagnosis. In addition, these older data do not reflect changes in survival and disease progression stemming from recent improvements to the treatment landscape. Although differences in the underlying populations and prevailing treatment regimens preclude direct comparisons, these earlier studies provide useful context for our tumor-specific findings. The reported cumulative incidence proportions at diagnosis and follow-up, respectively, were $7.6 \%$ and $12.1 \%$ for lung cancer (median follow-up 0.6 years), $1.5 \%$ and $5.8 \%$ for breast cancer (median follow-up 3.3 years), and 1.7\% and 5.9\% for prostate cancer (median follow-up 3.3 years).

Population-based estimates of the incidence of bone metastasis among patients with breast cancer have been reported for populations in Canada [19], the UK [18], and Denmark where survival after bone metastasis and related complications (SREs) has also been assessed [3, 7, 16, 20, 21] (Fig. 4). The Canadian study, which reports the experience of women diagnosed with nonmetastatic breast cancer between 1989 and 2001, reports on trends in the incidence of bone metastases over time. The 5-year incidence of bone metastasis underwent a continuous decrease $(7.46 \%$ [95\% CI: 6.66, 8.31], 5.25\% [95\% CI: 4.80, 5.71] and 3.54\% [95\% CI: 3.16, 3.96] in cohorts diagnosed between 1989-1991, 19921997, and 1998-2001. These cohorts were constructed to reflect important evolutions in the breast cancer treatment landscape. Specifically, in the first cohort, first generation CMF (cyclophosphamide, methotrexate, and 5 fluorouracil) chemotherapy without hormone therapy was used for premenopausal women with node positive cancers or high-risk node negative tumors. Postmenopausal women received tamoxifen regardless of tumor hormonal status, with those at high-risk also receiving 6 cycles of an anthracycline-containing regimen. The second cohort would have experienced increased tamoxifen use for premenopausal women and greater anthracyclinebased chemotherapy for both pre- and postmenopausal women. The third cohort would have seen greater use of adjuvant anthracyclines with the introduction of taxane and aromatase inhibitors in patients with either estrogen receptor positive $(E R+)$ or estrogen receptor negative (ER-) tumors.

In the UK study, the authors examined the experience of 13,207 women diagnosed with breast cancer between 2000 and 2006, using data from General Practice Research

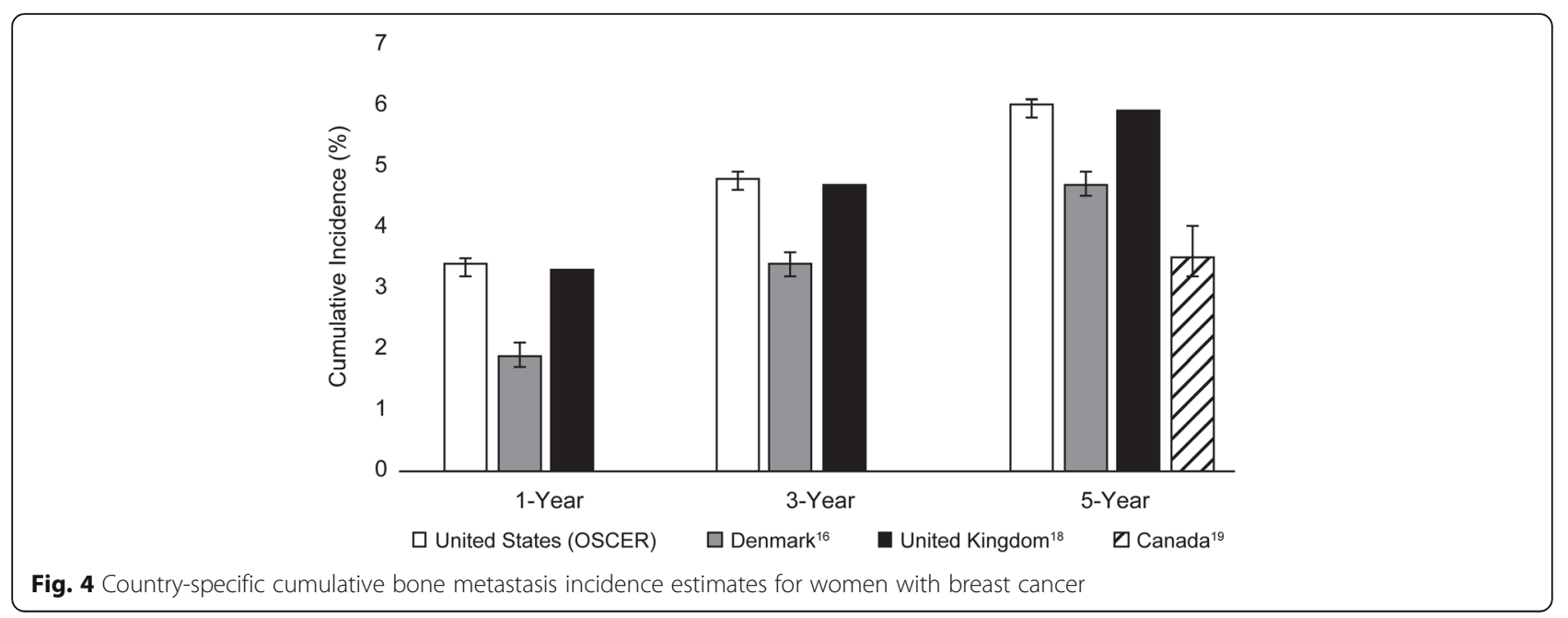


Database (GPRD) linked to the National Cancer Registry (NCR) and Hospital Episode Statistics (HES) [18]. In this population, most women had Stage 1 or 2 disease at diagnosis, but $2.6 \%$ of patients had metastatic breast cancer at diagnosis. After a median follow-up of 5.4 years, $6 \%$ of patients had developed bone metastases. The cumulative incidence of bone metastasis ranged from 3.3\% at one year to $5.9 \%$ at five years. Another smaller scale UK study examined the occurrence of distance metastases in women treated for primary invasive breast cancers at two National Health Service Trust Foundation hospitals between 1975 and 2006 [22]. The five year cumulative incidence of bone metastases was estimated at $6.9 \%$ (95\% CI, 6.3-7.5) among women with unilateral breast cancer, $11 \%$ (95\% CI, 5.1-16) among women with metachronous contralateral breast cancer occurring within five years of the initial diagnosis, and 2.3\% (95\% CI, 0.06-4.6) among women with metachronous contralateral breast cancer occurring more than five years after the initial diagnosis.

The population-based studies in Denmark used data from the Danish National Patient Registry (DNPR), which includes data from all hospitals in the country, to examine the incidence of bone metastases separately for patients with diagnosed with breast, lung and prostate cancer from 1999 through 2007. For a cohort of female breast cancer patients, Jensen et al. reported that the cumulative incidence of bone metastases increased from $1.9 \%(1.7-2.0)$ at one year to $3.4 \%(3.2-3.6)$ at three years to $4.7 \%(4.4-4.9)$ at five years [16]. In the prostate cancer cohort, the cumulative incidence of bone metastasis at one and five years after diagnosis was 7.7\% (7.4-8.1) and $16.6 \%$ (95\% CI 16.0-17.1), respectively [3]. In the lung cancer cohort, the cumulative incidence of bone metastases was 5.9\% (5.6-6.2) at one year and 6.7\% (6.4-7.0) at three years [20].

Development of bone metastases is an important prognostic indicator, with population-based studies demonstrating a significantly shorter survival after bone metastases occur. $[4-7,21]$ SREs may play an important role in the increased mortality risk subsequent to the development of bone metastases. Norgaard et al., for example, note that fewer than $1 \%$ of prostate cancer patients with bone metastases and SREs survived five years after their diagnosis [3], and suggest that SREs may signify more advanced or aggressive disease that shorten survival, and as other researchers have indicated [23], surgery for pathological fracture and loss of mobility and functional independence may also contribute to increased mortality [3].

Since 1996, three agents have been marketed in the US for the prevention of SREs in patients with bone metastasis secondary to solid tumors (intravenous bisphosphonates [IVBP]: zoledronic acid $(4 \mathrm{mg})$ and pamidronate disodium, dosed every 3-4 weeks; denosumab $120 \mathrm{mg}$, a RANK ligand inhibitor dosed every 4 weeks). With effective treatment options available and evidence regarding the significant mortality and morbidity implications of bone metastasis and SREs accumulating in the medical literature, bone health is increasingly addressed in key clinical guidelines [24, 25]. Even with this increased attention, one recent study of solid tumor patients with bone metastases in the US found that only $43 \%$ of commercially-insured patients and $47 \%$ of patients with Medicare coverage received bone targeted agents in 2012 [26]. Furthermore, over half of these patients (53\% commercial, 57\% Medicare) initiated these agents only after experiencing a bone complication. This finding is especially concerning in light of results from a recent study of breast cancer patients suggesting that the timing of bone targeting agent initiation has potential to significantly shape the level of therapeutic benefit to the patient [9]. In that study, the risk and frequency of SREs was higher if bone modifying agents (BMA) were not initiated until $\geq 6$ months after bone metastasis diagnosis. Additionally, the presence of extraskeletal metastases was associated with shorter time to first SRE.

Study limitations include access only to patients who received treatment or were under active surveillance at one of the OSCER-contributing clinics. Although this population includes patients with a variety of solid tumors, the tumor type distribution in our study differs from that in the U.S. population overall. Thus, the incidence estimate for the overall solid tumor category in our study may not be generalizable to the U.S. population. Specifically, patients with breast cancer may continue seeing their oncologists long after completing their active cancer treatment, and therefore, may be over-represented in the OSCER database. Prostate cancer patients overall, and early-stage patients in particular, may be under-represented in the study population, since many such patients are cared for exclusively at urology clinics. Estimates of bone metastasis incidence for all solid tumors combined are reported here for completeness and to provide context for the tumor-specific incidence estimates that we report. Our reliance on coded bone metastasis diagnoses may result in a conservative estimate of incidence. A recent study examining the validity of bone metastasis capture in the OSCER database found high specificity (98\%) and lower sensitivity (67\%) which provides reassurance that identified cases are true cases, yet suggests that identification of bone metastasis cases is not complete using the structured EMR data captured in OSCER [27]. Examination of the timing of bone metastasis coding suggested that the decision to treat (e.g., prescribing of a bone targeting agent or referral to orthopedic surgeon or radiation oncologist) may trigger the formal recording of a bone metastasis diagnosis. More 
generally, such misclassification is a limitation in all realworld databases used to estimate bone metastasis incidence [4-6], although the earlier validation study indicates that OSCER-based analyses are likely to better capture bone metastasis compared with analyses that use administrative claims data [28]. Ultimately, chart review remains the gold standard for case identification, but is feasable only for studies with small populations or limited followup, given the costs and records access required. Unlike such small-scale studies, our study provided access to EMR data for a large and diverse population of solid tumor patients in which we estimated the incidence of bone metastases during up to ten years of follow. In contrast to the potential underestimation associated with coding considerations, our incidence estimates include bone metastases that occurred around the index date (i.e., at index or within 30 days of index which can be interpreted as prevalent bone metastases) and this approach has the potential for overestimation. As expected, these early bone metastases were more likely to occur in patients with more advanced disease at diagnosis, and, although data on stage at diagnosis were limited (52\% missing) for the study population, half of the patients with bone metastases at or within 30 days of index were classified as Stage IV at diagnosis. Stage data is likely missing at random, since tumors are typically staged at the initial diagnosis, and these data are not routinely recorded in the structured portions of the electronic medical records. Although the true incidence of bone metastases may differ from our estimates, these results provide useful insights into bone metastasis occurrence and trends in the current treatment landscape.

\section{Conclusions}

In summary, our study estimated the incidence of bone metastases for solid tumor patients in the US, with 1-, 2-, 5- and 10-year estimates provided for solid tumors in aggregate, for individual tumor types, and by stage at diagnosis. Unique strengths of the study are the inclusion of all solid tumor types within a demographically and geographically broad population (no age or insurance type restrictions, a large population treated at over 52 oncology practices across the US), a follow-up period which is substantially longer than previously published incidence studies, and visibility into incidence shaped by current treatment approaches.

\footnotetext{
Abbreviations

BMA: bone modifying agents; Cl: confidence interval;

CMF: cyclophosphamide, methotrexate, and 5 fluorouracil; DNPR: Danish National Patient Registry; EMR: electronic medical records; ER: estrogen receptor; FDA: US Food and Drug Administration; GPRD: General Practice Research Database; HES: Hospital Episode Statistics; ICD-9: International Classification of Diseases, 9th Revision; IVBP: intravenous bisphosphonates; NCR: National Cancer Registry; OSCER: Oncology Services Comprehensive Electronic Records; SEER: Surveillance Epidemiology and End Results; SRE: skeletal-related event; UK: United Kingdom; US: United States
}

\section{Acknowledgements}

Initial study results were presented at the 2016 Annual Meeting of the American Society of Clinical Oncology [29]. The authors would also like to thank Dong Dai (IMS Health) for advising on and executing the statistical programming required for this study.

\section{Funding}

This study was sponsored by Amgen Inc. whose employees were also involved in designing the study, interpreting results, and writing the manuscript.

\section{Availability of data and materials}

The patient level dataset supporting the findings of this study will not be shared since permission for data-sharing was not obtained from all participating partners.

\section{Authors' contributions}

RKH, SW, AL, AR, MP, and GL collaborated to design the study. AR and MP were responsible for data access and analysis, and all authors collaborated to interpret results and develop the manuscript. All authors have read and approved the final version of this manuscript.

\section{Ethics approval and consent to participate}

The Institutional Review Board of each oncology practice approved collaboration to contribute data to a large longitudinal electronic health records database; informed patient consent was waived per the US framework for retrospective noninterventional studies. Individual patient-level data were protected against breach of confidentiality consistent with the final Health Insurance Portability and Accountability Act (HIPAA) Security Rule from the US Department of Health and Human Services.

\section{Consent for publication}

Not applicable.

\section{Competing interests}

RKH and AL are employees and stockholders of Amgen Inc. SW is employed by Wade Outcomes Research and Consulting which has conducted paid work for Amgen Inc. AR and MP are employees of IMS Health which has conducted paid work for Amgen Inc. GL is Principal Investigator on a research grant to the Fred Hutchinson Cancer Research Center from Amgen to study Febrile Neutropenia.

\section{Publisher's Note}

Springer Nature remains neutral with regard to jurisdictional claims in published maps and institutional affiliations.

\section{Author details \\ ${ }^{1}$ Amgen, Inc., One Amgen Center Drive, Thousand Oaks, CA 91320, USA. ${ }^{2}$ Wade Outcomes Research and Consulting, 358 South 700 East, Suite B432, Salt Lake City, UT 84102, USA. ${ }^{3}$ IMS Health, 1 IMS Drive, Plymouth Meeting, PA 19462, USA. ${ }^{4}$ Fred Hutchinson Cancer Research Center, University of Washington School of Medicine, 1100 Fairview Ave N, Seattle, Washington 98109, USA. ${ }^{5}$ Amgen, Inc., 1120 Veterans Blvd, South San Francisco, CA 94114, USA.}

Received: 29 June 2016 Accepted: 14 December 2017

Published online: 06 January 2018

\section{References}

1. Coleman RE, Lipton A, Roodman GD, Guise TA, Boyce BF, Brufsky AM, Clezardin P, Croucher PI, Gralow JR, Hadji P, et al. Metastasis and bone loss: advancing treatment and prevention. Cancer Treat Rev. 2010;36(8):615-20.

2. Li S, Peng Y, Weinhandl ED, Blaes AH, Cetin K, Chia VM, Stryker S, Pinzone JJ, Acquavella JF, Arneson TJ. Estimated number of prevalent cases of metastatic bone disease in the US adult population. Clin Epidemiol. 2012;4:87-93.

3. Norgaard M, Jensen AO, Jacobsen JB, Cetin K, Fryzek JP, Sorensen HT. Skeletal related events, bone metastasis and survival of prostate cancer: a population based cohort study in Denmark (1999 to 2007). J Urol. 2010; 184(1):162-7. 
4. Sathiakumar N, Delzell E, Morrisey MA, Falkson C, Yong M, Chia V, Blackburn J, Arora T, Brill I, Kilgore ML. Mortality following bone metastasis and skeletal-related events among women with breast cancer: a populationbased analysis of U.S. Medicare beneficiaries, 1999-2006. Breast Cancer Res Treat. 2012;131(1):231-8.

5. Sathiakumar N, Delzell E, Morrisey MA, Falkson C, Yong M, Chia V, Blackburn J, Arora T, Kilgore ML. Mortality following bone metastasis and skeletalrelated events among men with prostate cancer: a population-based analysis of US Medicare beneficiaries, 1999-2006. Prostate Cancer Prostatic Dis. 2011;14(2):177-83.

6. Sathiakumar N, Delzell E, Morrisey MA, Falkson C, Yong M, Chia V, Blackburn J, Arora T, Kilgore ML. Mortality following bone metastasis and skeletalrelated events among patients 65 years and above with lung cancer: a population-based analysis of U.S. Medicare beneficiaries, 1999-2006. Lung India : official Organ of Indian Chest Society. 2013;30(1):20-6.

7. Yong M, Jensen AO, Jacobsen JB, Norgaard M, Fryzek JP, Sorensen HT. Survival in breast cancer patients with bone metastases and skeletal-related events: a population-based cohort study in Denmark (1999-2007). Breast Cancer Res Treat. 2011;129(2):495-503.

8. Oster G, Lamerato L, Glass AG, Richert-Boe KE, Lopez A, Chung K, Richhariya A, Dodge T, Wolff GG, Balakumaran A, et al. Natural history of skeletalrelated events in patients with breast, lung, or prostate cancer and metastases to bone: a 15-year study in two large US health systems. Support Care Cancer. 2013;21(12):3279-86.

9. Tanaka R, Yonemori K, Hirakawa A, Kinoshita F, Takahashi N, Hashimoto J, Kodaira M, Yamamoto H, Yunokawa M, Shimizu C, et al. Risk factors for developing skeletal-related events in breast cancer patients with bone metastases undergoing treatment with bone-modifying agents. Oncologist. 2016;

10. Lipton A, Balakumaran A. Denosumab for the treatment of cancer therapyinduced bone loss and prevention of skeletal-related events in patients with solid tumors. Expert Rev Clin Pharmacol. 2012;5(4):359-71.

11. Roodman GD. Mechanisms of bone metastasis. N Engl J Med. 2004;350(16): 1655-64.

12. DePuy V, Anstrom KJ, Castel LD, Schulman KA, Weinfurt KP, Saad F. Effects of skeletal morbidities on longitudinal patient-reported outcomes and survival in patients with metastatic prostate cancer. Support Care Cancer. 2007;15(7):869-76.

13. Major PP, Cook R. Efficacy of bisphosphonates in the management of skeletal complications of bone metastases and selection of clinical endpoints. Am J Clin Oncol. 2002;25(6 Suppl 1):S10-8.

14. Barlev A, Song X, Ivanov B, Setty V, Chung K. Payer costs for inpatient treatment of pathologic fracture, surgery to bone, and spinal cord compression among patients with multiple myeloma or bone metastasis secondary to prostate or breast cancer. J Manag Care Pharm. 2010;16(9):693-702.

15. Coleman RE. Metastatic bone disease and the role of biochemical markers of bone metabolism in benign and malignant diseases. Cancer Treat Rev. 2001;27(3):133-5.

16. Jensen $A O$, Jacobsen JB, Norgaard $M$, Yong $M$, Fryzek JP, Sorensen HT. Incidence of bone metastases and skeletal-related events in breast cancer patients: a population-based cohort study in Denmark. BMC Cancer. 2011;11:29.

17. Sourbeer KN, Howard LE, Moreira DM, Amarasekara HS, Chow LD, Cockrell DC, Hanyok BT, Pratson CL, Kane CJ, Terris MK, et al. Practice patterns and predictors of followup imaging after a negative bone scan in men with castration resistant prostate cancer: results from the SEARCH database. J Urol. 2015;193(4):1232-8.

18. Hagberg KW, Taylor A, Hernandez RK, Jick S. Incidence of bone metastases in breast cancer patients in the United Kingdom: results of a multi-database linkage study using the general practice research database. Cancer Epidemiol. 2013;37(3):240-6.

19. Yerushalmi R, Woods R, Kennecke H, Speers C, Knowling M, Gelmon K. Patterns of relapse in breast cancer: changes over time. Breast Cancer Res Treat. 2010;120(3):753-9.

20. Cetin K, Christiansen CF, Jacobsen JB, Norgaard M, Sorensen HT. Bone metastasis, skeletal-related events, and mortality in lung cancer patients: a Danish population-based cohort study. Lung Cancer. 2014;86(2):247-54.

21. Cetin K, Christiansen CF, Svaerke C, Jacobsen JB, Sorensen HT. Survival in patients with breast cancer with bone metastasis: a Danish populationbased cohort study on the prognostic impact of initial stage of disease at breast cancer diagnosis and length of the bone metastasis-free interval. BMJ Open. 2015;5(4):e007702.

22. Vichapat V, Garmo H, Holmberg L, Fentiman IS, Tutt A, Gillett C, Luchtenborg M. Patterns of metastasis in women with metachronous contralateral breast cancer. Br J Cancer. 2012;107(2):221-3.

23. Oefelein MG, Ricchiuti V, Conrad W, Resnick MI. Skeletal fractures negatively correlate with overall survival in men with prostate cancer. J Urol. 2002; 168(3):1005-7.

24. Coleman R, Body JJ, Aapro M, Hadji P, Herrstedt J, Group EGW. Bone health in cancer patients: ESMO Clinical Practice Guidelines. Ann Oncol. 2014; 25(Suppl 3):iii124-37.

25. Gralow JR, Biermann JS, Farooki A, Fornier MN, Gagel RF, Kumar RN Shapiro CL, Shields A, Smith MR, Srinivas S, et al. NCCN Task Force Report: Bone Health in Cancer Care. J Natl Compr Canc Netw. 2009; 7(Suppl 3):S1-32; quiz S33-35

26. Hernandez RK, Adhia A, Wade SW, O'Connor E, Arellano J, Francis K, Alvrtsyan $\mathrm{H}$, Million RP, Liede A. Prevalence of bone metastases and bonetargeting agent use among solid tumor patients in the United States. Clin Epidemiol. 2015;7:335-45.

27. Liede A, Hernandez RK, Roth M, Calkins G, Larrabee K, Nicacio L. Validation of international classification of diseases coding for bone metastases in electronic health records using technology-enabled abstraction. Clin Epidemiol. 2015;7:441-8.

28. Hernandez RK, Quach D., Wade S W., Pirolli M., Reich A., Quigley J., Liede A.: Validation of Bone Metastases Coding in an Oncology Electronic Medical Records Database and a Commercial Claims Database. American College of Epidemiology Annual Meeting 2014.

29. Hernandez RK, Wade SW, Reich A, Pirolli MA, Liede A, Lyman GH: Incidence of boen metastases in U.S. patients with solid tumors. In: 2016 ASCO annual meeting: 2016; Chicago, IL; 2016.

\section{Submit your next manuscript to BioMed Central and we will help you at every step:}

- We accept pre-submission inquiries

- Our selector tool helps you to find the most relevant journal

- We provide round the clock customer support

- Convenient online submission

- Thorough peer review

- Inclusion in PubMed and all major indexing services

- Maximum visibility for your research

Submit your manuscript at www.biomedcentral.com/submit
) Biomed Central 